\title{
Fauna de morcegos em remanescentes urbanos de Cerrado em Campo Grande, Mato Grosso do Sul
}

\author{
Cláudia Márcia Marily Ferreira ${ }^{1,3}$, Erich Fischer ${ }^{2}$ \& Atenise Pulchério-Leite ${ }^{2}$ \\ ${ }^{1}$ Programa de Pós-graduação em Ecologia e Conservação, \\ Universidade Federal de Mato Grosso do Sul - UFMS, \\ CP 549, CEP 79070-900, Campo Grande, MS, Brasil \\ ${ }^{2}$ Departamento de Biologia, Universidade Federal de Mato Grosso do Sul-UFMS, \\ CP 549, CEP 79070-900, Campo Grande, MS, Brasil \\ ${ }^{3}$ Autora para correspondência: Cláudia Márcia Marily Ferreira, e-mail: claudiamarily@hotmail.com
}

FERREIRA, C.M.M., FISCHER, E. \& PULCHÉRIO-LEITE, A. Bat fauna in urban remnants of Cerrado in Campo Grande, Mato Grosso do Sul. Biota Neotrop. 10(3): http://www.biotaneotropica.org.br/v10n3/en/ abstract?article+bn02910032010.

\begin{abstract}
Campo Grande is the largest city of the State of Mato Grosso do Sul, located in the Cerrado domain, a biodiversity hotspot. Urban bat faunas have been poorly studied in Brazil, markedly in cities in the Cerrado region. The main objective here is describing composition, richness, and diversity of bat species which occur in the urban Cerrado remnants of Campo Grande. Bat samplings were carried out with mist-nets from March to August 2009 in eight urban parks. One sampling night was performed per month in each site, except in one; 47 nights at all. Each night, six $2.6 \times 12 \mathrm{~m}$ nets were kept open for six hours after sunset; the capture effort was $52.790 .4 \mathrm{~m}^{2} / \mathrm{h}$. We captured 701 bats belonging to 14 species of which 12 were phyllostomids $(98.6 \%$ of captures). Artibeus lituratus was the dominant species, such as reported in other urban regions in Brazil. Artibeus planirostris was the second most abundant species, in contrast to other urban bat assemblages. Chiroderma doriae, C. villosum, Phyllostomus hastatus e Platyrrhinus helleri were rare and registered for the first time in Campo Grande. Gathering other studies, 24 bat species are known to occur in Campo Grande, a high value among cities in the Cerrado. Bat diversity $\left(\mathrm{H}^{\prime}=1.65\right)$ in Campo Grande was also higher than that reported for other cities in the Cerrado or Atlantic Forest domains. The wide presence of forested parks in the urban zone may partially explain the great richness and diversity of bats in the Campo Grande city.
\end{abstract}

Keywords: Cerrado, Chiroptera, diversity, urban environment, sex ratio.

FERREIRA, C.M.M., FISCHER, E. \& PULCHÉRIO-LEITE, A. Fauna de morcegos em remanescentes urbanos de Cerrado em Campo Grande, Mato Grosso do Sul. Biota Neotrop. 10(3): http://www.biotaneotropica.org. br/v10n3/pt/abstract?article+bn02910032010.

Resumo: Campo Grande é a maior cidade do Estado de Mato Grosso do Sul, localizada no domínio do Cerrado, um hotspot de biodiversidade. A fauna de morcegos urbanos tem sido pouco estudada no Brasil, principalmente em cidades na região do Cerrado. O principal objetivo aqui é descrever a composição, a riqueza e a diversidade da fauna de morcegos em remanescentes de Cerrado na região urbana de Campo Grande. Amostragens de morcegos foram feitas entre março e agosto de 2009 em oito parques urbanos com auxílio de redes-de-neblina. Em cada local, exceto um, foi realizada uma noite de amostragem por mês; ao todo foram 47 noites. A cada noite, seis redes de 2,6 $\times 12 \mathrm{~m}$ foram mantidas abertas por seis horas a partir do ocaso; o esforço total de captura foi de $52.790,4 \mathrm{~m}^{2} / \mathrm{h}$. Foram capturados 701 morcegos pertencentes a 14 espécies, das quais 12 foram filostomídeos (98,6\% das capturas). Artibeus lituratus foi a espécie dominante, semelhante ao reportado para outras regiões urbanas no Brasil. Artibeus planirostris foi a segunda espécie mais abundante em Campo Grande, característica incomum em outras comunidades urbanas de morcegos. Chiroderma doriae, C. villosum, Phyllostomus hastatus e Platyrrhinus helleri foram raras, e registradas pela primeira vez em Campo Grande. Somados outros estudos, 24 espécies de morcegos foram encontradas em Campo Grande, valor alto dentre as cidades nos domínios do Cerrado. A diversidade $\left(H^{\prime}=1,65\right)$ de morcegos em Campo Grande também foi maior que a reportada para outras cidades em regiões de Cerrado ou Mata Atlântica. A ampla presença de parques florestados na zona urbana pode explicar, em parte, a grande riqueza e diversidade de morcegos na cidade de Campo Grande.

Palavras-chave: Cerrado, Chiroptera, diversidade, ambiente urbano, razão sexual. 


\section{Introdução}

O Cerrado apresenta grande diversidade biológica e muitas espécies endêmicas, sendo considerado um hotspot de biodiversidade (Myers et al. 2000). Entretanto, a biodiversidade do Cerrado está ameaçada pela expansão continuada de áreas para a agricultura, pastagens e pelo avanço de áreas urbanas (Klink \& Machado 2005). Atualmente, $60 \%$ da área do Cerrado apresentam estado original e apenas 5,2\% constituem áreas protegidas (Jepson 2005, Sano et al. 2007). Diante desse quadro, a manutenção da biodiversidade do Cerrado depende, em grande parte, do conhecimento e da conservação das espécies que habitam remanescentes florestais em áreas urbanas e rurais.

Os morcegos constituem o grupo mais diversificado de mamíferos em diversas regiões tropicais, onde desempenham importantes funções ecológicas nos sistemas naturais (Fleming et al. 1972, Reis et al. 2007). No Cerrado ocorrem pelo menos 103 espécies, o que representa aproximadamente $62 \%$ da fauna de morcegos do Brasil (Aguiar \& Zortéa 2008, Reis et al. 2007). Entretanto, apesar da grande diversidade deste grupo e da sua importância ecológica, o conhecimento atual sobre a fauna de morcegos do Cerrado é ainda insuficiente (Aguiar \& Zortéa 2008).

Diversas espécies de morcegos são capazes de ocupar áreas urbanas ou rurais, utilizando remanescentes de vegetação nativa, ou mesmo ambientes artificiais que ofereçam abrigos e fontes de alimentos (e.g. Sazima et al. 1994, Bredt \& Uieda 1996, Reis et al. 2002, Perini et al. 2003, Esbérard 2003, Lima 2008, Oprea et al. 2009). Algumas espécies encontram variedade e abundância de recursos alimentares e abrigos nas cidades, o que pode favorecer a ocorrência delas em regiões urbanas (Sazima et al. 1994, Bredt \& Uieda 1996, Silva et al. 1996, Reis et al. 2002, Lima 2008). Os objetivos deste trabalho são descrever a composição de espécies, a riqueza e a diversidade de morcegos, além de descrever a razão sexual e a proporção de jovens e adultos para as espécies de morcegos mais abundantes, em remanescentes de Cerrado na área urbana de Campo Grande.

\section{Material e Métodos}

Campo Grande ( $20^{\circ} 27^{\prime} \mathrm{S}$ e $\left.54^{\circ} 37^{\prime} \mathrm{O}\right)$ é a capital e a maior cidade ( 750 mil habitantes) do Estado de Mato Grosso do Sul, Centro-oeste do Brasil. O município abrange aproximadamente $8.100 \mathrm{~km}^{2}$, com área urbana de $150 \mathrm{~km}^{2}$ e topografia plana (590 m altitude). Está localizada no divisor das bacias hidrográficas do Paraná e do Paraguai, com grande quantidade de córregos e nascentes. O clima é tropical (Aw de Köppen), com inverno seco e verão úmido; a precipitação média anual é de $1.500 \mathrm{~mm}$ e a temperatura média anual de $23^{\circ} \mathrm{C}$ (Coleti et al. 2007). Espécies da vegetação original de Cerrado predominam em parques e remanescentes urbanos de vegetação nativa. Aproximadamente $30 \%$ da área do município correspondem à vegetação remanescente de Cerrado (Instituto Municipal de Planejamento Urbano 2007).

Oito remanescentes urbanos de Cerrado foram selecionados em Campo Grande (Figura 1): Parque Estadual do Prosa (142,0 ha), cuja vegetação apresenta formações de cerrado sentido restrito, cerradão e floresta semidecídua (mata ciliar); Parque Florestal Antônio Albuquerque (2,5 ha), que abriga espécies de árvores do Cerrado e exóticas; Parque Ecológico Alexandre Rodrigues Ferreira (23,6 ha), com vegetação típica de cerrado sentido restrito; Parque Estadual Matas do Segredo (172,0 ha), principalmente coberto por cerrado sentido restrito, mas também apresenta florestas semidecíduas e florestas inundáveis em regiões de nascentes; Estação Ecológica Dahma (42,3 ha), cujas fisionomias presentes são cerradão e floresta semidecídua; Reserva Biológica da Universidade Federal de Mato Grosso do Sul (35,6 ha) que apresenta vegetação de cerrado sentido restrito e cerradão; Reserva Florestal da Base Aérea (29,0 ha), pertencente à aeronáutica, foi originalmente coberta por veredas que atualmente estão descaracterizadas, além de porções de cerrado sentido restrito que sofreram queimadas e corte seletivo de árvores (Coleti et al. 2007); Mata da Universidade Católica Dom Bosco (32,1 ha) que apresenta vegetação de cerrado sentido restrito e cerradão.

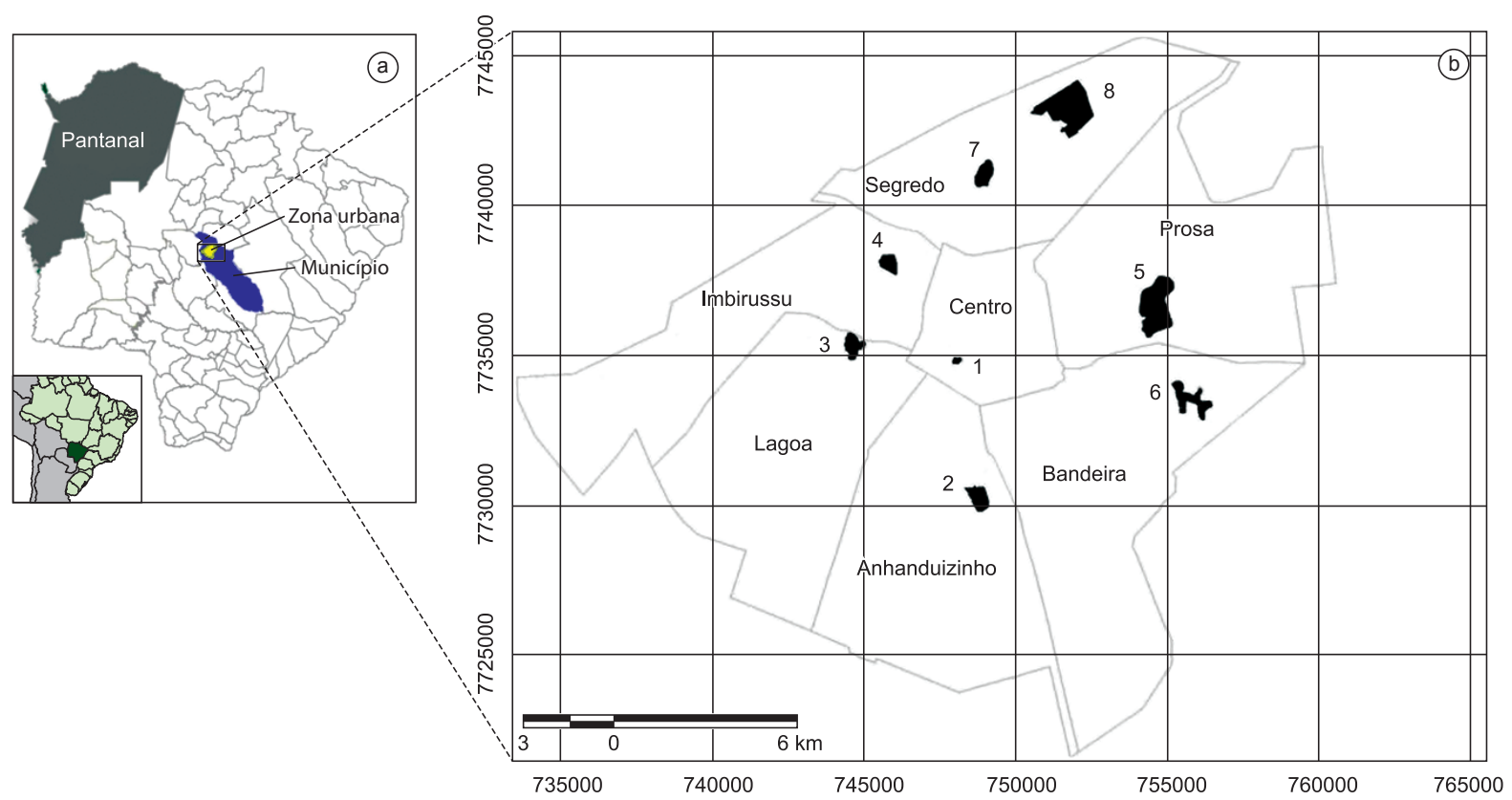

Figura 1. a) Limites territoriais dos municípios de Mato Grosso do Sul, Brasil, com destaque para a área do município (azul) e a zona urbana (amarelo) de Campo Grande; b) subdivisões da zona urbana de Campo Grande e localização dos remanescentes estudados: 1)Parque Florestal Antônio Albuquerque; 2) Reserva Biológica da Universidade Federal de Mato Grosso do Sul; 3) Reserva Florestal da Base Aérea; 4) Parque Ecológico Alexandre Rodrigues Ferreira; 5) Parque Estadual do Prosa; 6) Estação Ecológica Dahma; 7) Mata da Universidade Católica Dom Bosco; 8) Parque Estadual Matas do Segredo.

Figure 1. a) Territorial limits of municipalities of Mato Grosso do Sul, Brazil, with territorial area (blue) and urban zone (yellow) of Campo Grande highlighted; b) Subdivisions of Campo Grande urban zone and location of the studied remnants: 1)Parque Florestal Antônio Albuquerque; 2) Reserva Biológica da Universidade Federal de Mato Grosso do Sul; 3) Reserva Florestal da Base Aérea; 4) Parque Ecológico Alexandre Rodrigues Ferreira; 5) Parque Estadual do Prosa; 6) Estação Ecológica Dahma; 7) Mata da Universidade Católica Dom Bosco; 8) Parque Estadual Matas do Segredo. 
As espécies de morcegos foram amostradas mensalmente de março a agosto de 2009, totalizando 47 noites. Em cada remanescente foram feitas seis noites de amostragens, uma vez por mês, exceto no Parque Estadual do Prosa onde foram cinco noites de amostragem. A cada noite, os morcegos foram capturados com auxílio de seis redes-de-neblina $(2,6 \times 12,0 \mathrm{~m})$ que permaneceram abertas durante seis horas a partir do ocaso. As redes foram fixadas entre 0,5 e $3,1 \mathrm{~m}$ acima do solo, em trilhas ou clareiras no interior da mata. $\mathrm{O}$ esforço de captura, medido como a área $\left(\mathrm{m}^{2}\right)$ total das redes multiplicada pelo tempo (horas) de exposição (cf. Straube \& Bianconi 2002), foi de $52.790,4 \mathrm{~m}^{2} / \mathrm{h}$, divididos em $5.616 \mathrm{~m}^{2} / \mathrm{h}$ no Parque Estadual do Prosa e $6.739,2 \mathrm{~m}^{2} / \mathrm{h}$ para cada uma das demais áreas. Os morcegos capturados foram inicialmente mantidos em sacos de pano individuais e posteriormente manipulados para identificação da espécie (cf. Vizotto \& Taddei 1973 modificado por W. Uieda, comunicação pessoal; Gregorin \& Taddei 2002) e registros do sexo (cf. Racey 1988), da classe etária (jovem ou adulto) (cf. Anthony 1988), do comprimento do antebraço (precisão de $0,1 \mathrm{~mm}$ ) e da massa (precisão de $1 \mathrm{~g}$ ). Em seguida, os indivíduos receberam um colar com anilha numerada para identificação de recapturas e foram soltos. Indivíduos foram coletados para confirmação da identificação das espécies e depositados como material testemunho na Coleção Zoológica (ZUFMS-M) da Universidade Federal de Mato Grosso do Sul (sob números 491 a 504; 506 a 508).

Para avaliar a suficiência do esforço amostral foi construída curva de rarefação com auxílio do programa Past (Hammer et al. 2001). Este programa também foi usado para cálculo do índice de diversidade de Shannon (H') e intervalos de confiança (95\%) (Bootstrap, 1000 repetições), assim como para avaliar desvios da curva de ranking-abundância das espécies com respeito aos modelos (cf. Magurran 2004): série geométrica, série logarítmica, série normal logarítmica e bastão quebrado ("broken stick"). Para estimar a riqueza de espécies esperada foi utilizado o estimador Chao 2 (1000 repetições) com auxílio do programa EstimateS (Colwell 2005). Para seis espécies representadas por maior quantidade $(\geq 20)$ de indivíduos capturados foram avaliados desvios da razão sexual 1:1 e da proporção entre adultos e jovens com uso de Teste de Quiquadrado para proporções esperadas iguais, e auxílio do programa BioEstat (Ayres et al. 2007). As demais espécies foram representadas por quantidade $(\leq 7)$ de indivíduos insuficiente para avaliar desvios da razão sexual ou entre classes de idade.

\section{Resultados}

Foram capturados 701 indivíduos pertencentes a 14 espécies, 10 gêneros e três famílias (Tabela 1). Phyllostomidae foi a família predominante, representada por 12 espécies e 98,6\% das capturas. As espécies mais capturadas foram Artibeus lituratus (Olfers 1818), Artibeus planirostris (Spix 1823), Platyrrhinus lineatus (E. Geoffroy 1810) e Carollia perspicillata (Linnaeus 1758) (Tabela 1). Juntas, estas quatro espécies representaram $87 \%$ das capturas $(n=610)$. Três espécies (21\%) ocorreram em todas as áreas amostradas: A. lituratus, A. planirostris e P. lineatus. Outras espécies de filostomídeos Anoura caudifer (E. Geoffroy 1818), Chiroderma doriae (Thomas 1891), Chiroderma villosum (Peters 1860), Phyllostomus discolor (Wagner 1843), Phyllostomus hastatus (Pallas 1767) e Platyrrhinus helleri (Peters 1866) - foram relativamente raras em nossas amostras. Morcegos molossídeos e vespertilionídeos também foram pouco capturados, corresponderam a 1,4\% dos indivíduos (Tabela 1).

A curva de rarefação atingiu assíntota e mostrou tendência de leve incremento de espécies com o aumento do esforço de capturas (Figura 2). A riqueza total de morcegos estimada (Chao 2) para os remanescentes urbanos de Campo Grande foi igual a 15 espécies,
Tabela 1. Número de indivíduos machos (J: jovens, A: adultos) e fêmeas (J: jovens, NR: adultas não-reprodutivas, G: grávidas, L: lactantes) de 14 espécies de morcegos capturadas em remanescentes florestais urbanos de Campo Grande, Mato Grosso do Sul.

Table 1. Number of male (J: young, A: adults) and female (J: young, NR: adult no-reproductive, G: pregnant, L: lactic) individuals of 14 species of bats captured in urban forest remnants of Campo Grande, Mato Grosso do Sul.

\begin{tabular}{|c|c|c|c|c|c|c|c|c|c|}
\hline \multirow{2}{*}{$\begin{array}{l}\text { Família } \\
\text { Espécie }\end{array}$} & \multicolumn{3}{|c|}{ Machos } & \multicolumn{5}{|c|}{ Fêmeas } & \multirow[t]{2}{*}{$\mathbf{N}^{\mathbf{a}}$} \\
\hline & $\mathbf{J}$ & $\mathbf{A}$ & $\sum$ & $\mathbf{J}$ & NR & G & $\mathbf{L}$ & $\sum$ & \\
\hline \multicolumn{10}{|l|}{ Phyllostomidae } \\
\hline Artibeus lituratus ${ }^{\mathrm{b}}$ & 55 & 89 & 144 & 62 & 90 & 3 & 2 & 157 & 301 \\
\hline Artibeus planirostris ${ }^{\mathrm{c}}$ & 30 & 36 & 66 & 34 & 56 & 5 & 7 & 102 & 168 \\
\hline Platyrrhinus lineatus ${ }^{\mathrm{d}}$ & 7 & 20 & 27 & 11 & 30 & 2 & 1 & 44 & 71 \\
\hline Carollia perspicillata & 4 & 17 & 21 & 12 & 36 & 1 & 0 & 49 & 70 \\
\hline Glossophaga soricina & 0 & 12 & 12 & 9 & 17 & 0 & 1 & 27 & 39 \\
\hline Sturnira lilium ${ }^{\mathrm{f}}$ & 1 & 5 & 6 & 2 & 11 & 2 & 0 & 15 & 21 \\
\hline Phyllostomus discolor & 3 & 2 & 5 & 0 & 2 & 0 & 0 & 2 & 7 \\
\hline Chiroderma doriae & 0 & 5 & 5 & 0 & 1 & 0 & 0 & 1 & 6 \\
\hline Anoura caudifer & 0 & 3 & 3 & 0 & 0 & 1 & 0 & 1 & 4 \\
\hline Platyrrhinus helleri & 0 & 0 & 0 & 2 & 0 & 0 & 0 & 2 & 2 \\
\hline Chiroderma villosum & 0 & 0 & 0 & 1 & 0 & 0 & 0 & 1 & 1 \\
\hline Phyllostomus hastatus & 1 & 0 & 1 & 0 & 0 & 0 & 0 & 0 & 1 \\
\hline \multicolumn{10}{|l|}{ Vespertilionidae } \\
\hline Myotis nigricans & 1 & 2 & 3 & 0 & 4 & 0 & 0 & 4 & 7 \\
\hline \multicolumn{10}{|l|}{ Molossidae } \\
\hline Molossops temminckii & 0 & 1 & 1 & 0 & 2 & 0 & 0 & 2 & 3 \\
\hline
\end{tabular}

${ }^{\mathrm{a}}$ Recapturas não foram somadas; ${ }^{\mathrm{b}, \mathrm{c}}$ seis recapturas; ${ }^{\mathrm{d}}$ duas recapturas; ${ }^{\mathrm{e}}$ três recapturas, ${ }^{\mathrm{f}}$ uma recaptura.

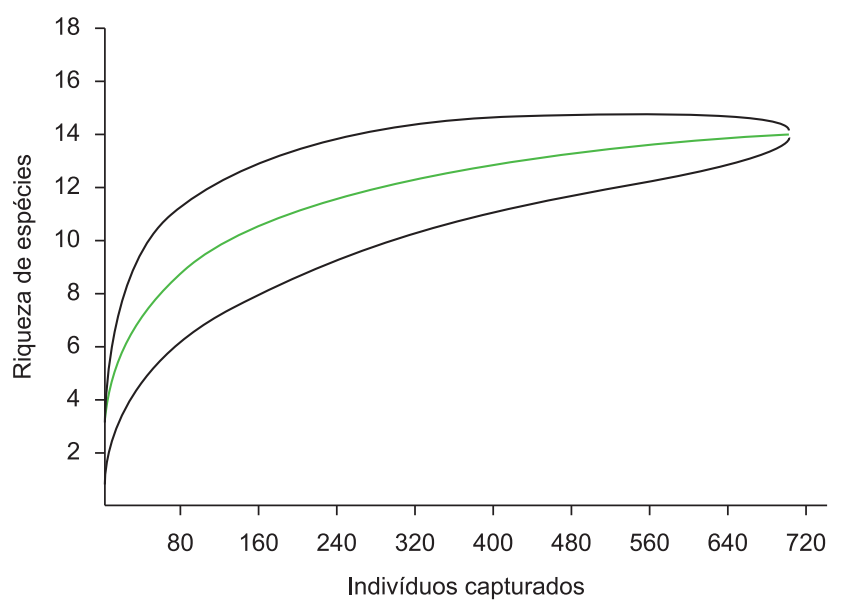

Figura 2. Curva de rarefação (verde) para espécies de morcegos amostradas em remanescentes urbanos de Cerrado em Campo Grande, Mato Grosso do Sul (linhas pretas = IC 95\%).

Figure 2. Rarefaction curve (green) for bat species surveyed in urban remnants of Cerrado in Campo Grande, Mato Grosso do Sul (black lines $=95 \% \mathrm{CI}$ ).

indicando que a amostragem realizada aqui incluiu $93 \%$ das espécies de morcegos esperadas, cuja ocorrência é possível de registro por meio de redes-de-neblina. A curva de ranking-abundância das espécies (Figura 3 ) não diferiu $\left(\chi^{2}=15,01 ; \mathrm{p}=0,09\right)$ do modelo série logarítmica, porém diferiu dos modelos série geométrica $\left(\chi^{2}=58,74\right.$; $\mathrm{p}<0,0001)$, série normal logarítmica $\left(\chi^{2}=3,71 ; \mathrm{p}<0,05\right)$ e bastão quebrado $\left(\chi^{2}=273,50 ; \mathrm{p}<<0,0001\right)$. O índice de diversidade $\left(\mathrm{H}^{\prime}\right)$ foi de 1,65 (95\% IC entre 1,56 e 1,72). 


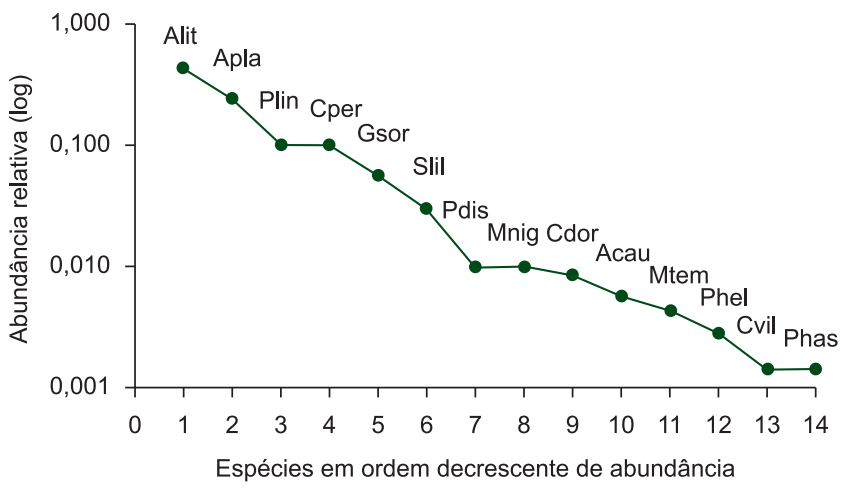

Figura 3. Curva de ranking-abundância (abundância das espécies de morcegos pelas espécies em ordem decrescente de abundância), em remanescentes urbanos de Campo Grande, Mato Grosso do Sul. Alit - Artibeus lituratus; Apla - Artibeus planirostris; Plin - Platyrrhinus lineatus; Cper - Carollia perspicillata; Gscr - Glossophaga soricina; Slil- Sturnira lilium; Pdis - Phyllostomus discolor; Mnig - Myotis nigricans; Cdor - Chiroderma doriae; Acau - Anoura caudifer; Mtem - Molossops temminckii; Phel-Platyrrhinus helleri; Cvil-Chiroderma villosum; Phas - Phyllostomus hastatus.

Figure 3. Rank-abundance curve (abundance of bat species on species in decreasing order of abundance), in urban remnants of Campo Grande, Mato Grosso do Sul. Alit - Artibeus lituratus; Apla - Artibeus planirostris; Plin - Platyrrhinus lineatus; Cper - Carollia perspicillata; Gscr - Glossophaga soricina; Slil- Sturnira lilium; Pdis - Phyllostomus discolor; Mnig-Myotis nigricans; Cdor-Chiroderma doriae; Acau - Anoura caudifer; Mtem - Molossops temminckii; Phel - Platyrrhinus helleri; Cvil - Chiroderma villosum; Phas - Phyllostomus hastatus.

Dentre as seis espécies com mais indivíduos capturados, cinco apresentaram razão sexual desviada para fêmeas - Sturnira lilium (E. Geoffroy 1810) (2,5 fêmeas:1 macho; $\left.\chi^{2}=3,86 ; \mathrm{p}<0,05\right)$, C. perspicillata (2,3 fêmeas:1 macho; $\left.\chi^{2}=11,20 ; \mathrm{p}<0,001\right)$, Glossophaga soricina (Pallas 1766) (2,3 fêmeas: 1 macho; $\chi^{2}=5,77$; $\mathrm{p}<0,02)$, P. lineatus (1,6 fêmeas: 1 macho; $\left.\chi^{2}=4,07 ; \mathrm{p}<0,05\right)$ e A. planirostris ( 1,5 fêmeas: 1 macho; $\left.\chi^{2}=7,71 ; \mathrm{p}<0,01\right)$. Entretanto, a espécie mais comum - A. lituratus - não apresentou razão sexual diferente de $1: 1\left(\chi^{2}=0,56 ; p=0,49\right)$. Quanto às classes de idade, estas seis espécies de morcegos apresentaram maior proporção de indivíduos adultos $-S$. lilium (6 adultos:1 jovem; $\chi^{2}=10,71$; $\mathrm{p}<0,01)$, C. perspicillata $\left(3,4\right.$ adultos: 1 jovem; $\chi^{2}=20,63$; $\mathrm{p}<0,0001), G$. soricina $\left(3,3\right.$ adultos: 1 jovem; $\chi^{2}=11,31$; $\mathrm{p}<0,001)$, P. lineatus (2,9 adultos: 1 jovem; $\left.\chi^{2}=17,25 ; \mathrm{p}<0,0001\right)$, A. planirostris $\left(1,6\right.$ adultos: 1 jovem; $\left.\chi^{2}=9,52 ; \mathrm{p}<0,02\right)$ e $A$. lituratus (1,6 adultos: 1 jovem; $\left.\chi^{2}=14,91 ; \mathrm{p}<0,0001\right)$.

\section{Discussão}

A predominância de morcegos filostomídeos nos remanescentes urbanos de Campo Grande é um resultado esperado devido ao uso de redes-de-neblina e à grande riqueza desta família em toda região neotropical (Fenton et al. 1992). Morcegos vespertilionídeos parecem ser hábeis em detectar as redes, e molossídeos geralmente forrageiam acima das copas (Pedro \& Taddei 1997), por isso são grupos provavelmente subamostrados aqui. Apesar da baixa frequência de captura neste estudo, molossídeos e vespertilionídeos são comuns em ambientes urbanos e não estão necessariamente associados a remanescentes de vegetação natural; muitas vezes forrageiam insetos atraídos pela iluminação de casas e ruas e abrigam-se em edificações humanas (e.g. Silva et al. 1996, Reis et al. 2002, Perini et al. 2003, Fischer et al. 2010).
Em Campo Grande, a manutenção de remanescentes da vegetação original e a presença de plantas exóticas exploradas por morcegos na arborização urbana provavelmente favorece espécies herbívoras, como A. lituratus, A. planirostris, P. lineatus, C. perspicillata, G. soricina, S. lilium, C. doriae, C. villosum e P. helleri. Plantas como Terminalia catappa L. (Combretaceae) (amendoeira), Syzygium jambolanum (Lam.) DC. (Myrtaceae) (jamelão), bem como espécies de Ficus L. (Moraceae) (figueira), Cecropia Tréc. (Urticaceae) (embaúba), Piper L. (Piperaceae) e Bauhinia L. (Fabaceae) (pata-de-vaca), são comuns em Campo Grande (obs. pess.) e amplamente utilizadas por espécies de filostomídeos em diversas cidades e ambientes naturais (Sazima et al. 1994, Bredt \& Uieda 1996, Morais 2002, Perini et al. 2003, Aguiar \& Marinho-Filho 2007, Teixeira et al. 2009). Artibeus lituratus é a espécie mais abundante em Campo Grande, semelhante ao encontrado em algumas cidades estudadas (e.g. Sazima et al. 1994, Barros et al. 2006, Oprea et al. 2009). O sucesso desta espécie em áreas urbanas se deve provavelmente ao comportamento oportunista, ao hábito de abrigar-se em grupos pequenos na vegetação e à dieta variada - que inclui frutos, pólen, néctar, folhas e insetos (Sazima et al. 1994, Zortéa \& Chiarello 1994, Fischer, W.A. \& Fischer, E.A., Bredt \& Uieda 1996, De Knegt et al. 2005). Dentre as demais espécies - A. planirostris, P. lineatus, C. perspicillata, G. soricina e S. lilium - abundantes nos fragmentos estudados, apenas A. planirostris não é comum em outras regiões urbanas estudadas (Bredt \& Uieda 1996, Silva et al. 1996, Esbérard 2003, Perini et al. 2003, De Knegt et al. 2005, Ortêncio-Filho et al. 2005, Lima 2008, Oprea et al. 2009). A destacada abundância de A. planirostris em Campo Grande comparativamente a outras cidades pode estar relacionada à aparente maior densidade desta espécie na porção sul dos domínios do Cerrado e do Pantanal (Bordignon 2006, Camargo et al. 2009, Cunha et al. 2009, Teixeira et al. 2009), região onde Campo Grande está inserida.

Os filostomídeos relativamente raros nos remanescentes estudados $-A$. caudifer, C. doriae, $C$. villosum, P. helleri, $P$. discolor e $P$. hastatus - têm sido também reportados como pouco abundantes em diferentes locais (e.g. Bianconi et al. 2004, Bordignon 2006, Cáceres et al. 2008, Lima 2008, Camargo et al. 2009); entretanto, P. discolor foi uma das espécies mais abundantes em locais da Amazônia e da Mata Atlântica (Bernard \& Fenton 2002, Faria \& Baumgarten 2007). $\mathrm{O}$ fato de $P$. discolor e $P$. hastatus apresentarem dieta marcadamente generalista (Santos et al. 2003, Kwiecinski 2006) deve favorecer a ocorrência destas espécies em ambiente urbano. Porém, são espécies de morcegos grandes que demandam ampla área de forrageamento e, por isso, poderiam apresentar populações com baixas densidades (Fleming et al. 1972, Purvis et al. 2000). Adicionalmente, P. discolor e $P$. hastatus forrageiam predominantemente nos estratos mais altos da vegetação (Fischer 1992, Bernard 2001), comportamento que reduz a probabilidade de capturas em redes dispostas ao nível do solo. Chiroderma doriae e C. villosum apresentam dieta aparentemente muito especializada em consumo de espécies silvestres de Ficus (Nogueira \& Peracchi 2003), característica desfavorável em ambientes alterados. A ocorrência de espécies que demandam áreas amplas ou que apresentam hábito alimentar especializado sustenta que os remanescentes urbanos de Campo Grande mantêm espécies pouco favorecidas, ou mesmo desfavorecidas, em outros ambientes perturbados. Mais dados sobre a biologia das espécies de morcegos, bem como sobre os recursos e condições disponíveis nos remanescentes estudados, são necessários para conhecer os fatores que determinam os resultados encontrados aqui.

As espécies registradas aqui somadas a outros registros disponíveis (Pulchério-Leite et al. 1999, Deus et al. 2003) sustentam a ocorrência de pelo menos 24 espécies de morcegos na cidade 
de Campo Grande - 13 filostomídeos, cinco molossídeos e seis vespertilionídeos. Este valor representa $23 \%$ das espécies de morcegos conhecidas para o Cerrado e 39\% das espécies reportadas para Mato Grosso do Sul (Aguiar \& Zortéa 2008, Cáceres et al. 2008, Cunha et al. 2009). A riqueza de morcegos na região urbana de Campo Grande, assim como de Cuiabá ( $\mathrm{n}=26$ espécies; Morais 2002), pode ser considerada alta quando comparada com outras regiões urbanas em domínio de Cerrado. Apenas 17 espécies foram registradas em Brasília (Bredt \& Uieda 1996), 16 espécies em Belo Horizonte (Perini et al. 2003) e 13 espécies em Uberlândia (Stutz et al. 2004). Uma possível explicação para a grande riqueza de morcegos em Campo Grande pode ser a ampla presença de áreas verdes na cidade (Coleti et al. 2007). A diversidade ( $\left.H^{\prime}\right)$ de espécies nos remanescentes urbanos de Campo Grande $(1,65)$ também pode ser considerada alta quando comparada a outras cidades nos domínios do Cerrado, como Belo Horizonte (1,30; De Knegt et al. 2005), e da Mata Atlântica, como Cianorte (1,33; Ortêncio-Filho et al. 2005), Juiz de Fora (0,90; Barros et al. 2006), Blumenau (1,51; Gruener 2006) e Vitória (1,41; Oprea et al. 2009). O melhor ajuste da curva de ranking-abundância com o modelo série logarítmica indica que a dominância das espécies mais comuns sobre as menos abundantes pode ser considerada de moderada a alta (Magurran 2004) nos remanescentes urbanos de Campo Grande. Este padrão de distribuição de abundância relativa entre as espécies pode ser determinado por competição interespecífica (Stevens \& Willig 2000), nesse caso as espécies mais comuns seriam competitivamente superiores.

Maior proporção de fêmeas em relação aos machos tem sido observada para espécies de morcegos filostomídeos (e.g. Mello \& Fernandez 2000, Zortéa 2003, Costa et al. 2007, Mello et al. 2009). Muitas espécies apresentam sistema de acasalamento poligínico, com a formação de haréns organizados a partir de machos adultos (Kunz et al. 1983, Fleming 1992, Fischer, W.A. \& Fischer, E.A., Zortéa 2003, Mello et al. 2009). Machos polígamos podem ser menos capturados devido à necessidade de passarem proporcionalmente mais tempo defendendo seus territórios e abrigos do que forrageando (Fischer, W.A. \& Fischer, E.A., Pulchério-Leite 2008). Embora A. lituratus apresente grupos estruturados sob a forma de haréns, a razão entre fêmeas e machos não diferiu de 1:1. Este resultado pode estar relacionado à maior proporção de indivíduos jovens de A. lituratus, cujos machos não constituíram haréns. As espécies mais abundantes, A. lituratus e A. planirostris, apresentam menor desproporção entre adultos e jovens, indicando que são espécies com maior recrutamento na cidade de Campo Grande durante o período de estudo. De modo geral, a maior proporção de adultos que jovens pode ser explicada pela baixa quantidade de filhotes por ano (um a dois por fêmea), comum a muitas espécies de morcegos filostomídeos (e.g. Fleming et al. 1972, Mello \& Fernandez 2000).

\section{Agradecimentos}

Ao Instituto de Meio Ambiente de Mato Grosso do Sul (IMASUL) e a Secretaria Municipal de Meio Ambiente e Desenvolvimento Sustentável (SEMADES) pelas autorizações para o desenvolvimento desse estudo nas Unidades de Conservação; ao tenente coronel Aron Zimmermann, coronel Eduardo Pantoja, Elza Mara e Emerson Pistori pelas autorizações e apoio para atividades de campo, respectivamente, na Base Aérea, Colégio Militar, Horto Florestal e Universidade Católica Don Bosco (UCDB); à Carolina dos Santos pelo auxílio na identificação das espécies; à FUNDECT, Earthwatch Institute e PROPP-UFMS pelo financiamento; à CAPES, CNPq e FUNDECT pela concessão de bolsas para C.M.M. Ferreira, E. Fischer e A. Pulchério-Leite, respectivamente.

\section{Referências Bibliográficas}

AGUIAR, L.M.S. \& MARINHO-FILHO, J. 2007. Bat frugivory in a remnant of Southeastern Brazilian Atlantic forest. Acta Chiropt. 9(1):251-260.

AGUIAR, L.M.S. \& ZORTÉA, M. 2008. A diversidade de morcegos conhecida para o Cerrado. In Simpósio Nacional Cerrado \& Simpósio Internacional Savanas Tropicais, Brasília, DF. Desafios e estratégias para o equilíbrio entre sociedade, agronegócio e recursos naturais: anais. Embrapa Cerrados, Planaltina, DF.

ANTHONY, E.L.P. 1988. Age determination in bats. In Ecological and behavioral methods for the study of bats (T.H. Kunz, ed.). Smithsonian Institution, Washington, p.47-58.

AYRES, M., AYRES Jr., M., AYRES, D.L. \& SANTOS, A.A.S. 2007. BioEstat: aplicações estatísticas nas áreas das ciências bio-médicas. 5 ed. Pará, Brasil.

BARROS, R.S.M., BISAGGIO, E.L. \& BORGES, R.C. 2006. Morcegos (Mammalia, Chiroptera) em fragmentos florestais urbanos no município de Juiz de Fora, Minas Gerais, Sudeste do Brasil. Biota Neotrop. 6: http://www. biotaneotropica.org.br/v6n1/pt/abstract?inventory+bn02206012006.

BERNARD, E. 2001. Vertical stratification of bat communities in primary forests of Central Amazon, Brazil. J. Trop. Ecol. 17:115-126.

BERNARD, E. \& FENTON, M.B. 2002. Species diversity of bats (Mammalia: Chiroptera) in forest fragments, primary forests, and savannas in central Amazonia, Brazil. Can. J. Zool. 80:1124-1140.

BIANCONI, G.V., MIKICH, S.B. \& PEDRO, W.A. 2004. Diversidade de morcegos (Mammalia, Chiroptera) em remanescentes florestais do município de Fênix, noroeste do Paraná. Brasil. Rev. Bras. Zool. 21(4):943-954.

BORDIGNON, M.O. 2006. Diversidade de morcegos (mammalian, chiroptera) do complexo Aporé-Sucuriú, Mato Grosso do Sul, Brasil. Rev. Bras. Zool. 23(4):1002-1009.

BREDT, A. \& UIEDA, W. 1996. Bats from urban and rural environments of the Distrito Federal, mid-western Brazil. Chiropt. Neotrop. 2:54-57.

CÁCERES, N.C., CARMIGNOTTO, A.P., FISCHER, E. \& SANTOS, C.F. 2008. Mammals from Mato Grosso do Sul, Brazil. Check List 4(3):321-335.

CAMARGO, G., FISCHER, E., GONÇALVES, F., FERNANDES, G. \& FERREIRA, S. 2009. Morcegos do Parque Nacional da Serra da Bodoquena, Mato Grosso do Sul, Brasil. Chiropt. Neotrop. 15(1):417-424.

COLETI, R.C.F.B., LUCHMANN, R. \& DAMBRÓS, S.R. 2007. Relatório de avaliação ambiental. Programa de desenvolvimento integrado e qualificação urbana do município de Campo Grande MS. Prefeitura Municipal de Campo Grande, Campo Grande.

COLWELL, R.K. 2005. EstimateS. Statistic estimation of species richness and shared species from samples. Version 7.5. User's Guide and application. http://purloclc.org/estimates

COSTA, L.M., ALMEIDA, J.C. \& ESBÉRARD, C.E.L. 2007. Dados de reprodução de Platyrrhinus lineatus em estudo de longo prazo no Estado do Rio de Janeiro (Mammalia, Chiroptera, Phyllostomidae). Iheringia, Sér. Zool. 97(2):152-156.

CUNHA, N.L., FISCHER, E., CARVALHO, L.F.A.C. \& SANTOS, C.F 2009. Bats of Buraco das Araras natural reserve, southwestern Brazil. Biota Neotrop. 9: http://www.biotaneotropica.org.br/v9n4/en/abstract?i nventory+bn02909042009.

DE KNEGT, L.V., SILVA, J.A., MOREIRA, E.C. \& SALES, G.L. 2005. Morcegos capturados no município de Belo Horizonte, 1999-2003. Arq. Bras. Med. Vet. Zootec. 57:576-583.

DEUS, G.T., BECER, M. \& NAVARRO, I.T. 2003. Diagnóstico da raiva em morcegos não hematófagos na cidade de Campo Grande, Mato Grosso do Sul, Centro Oeste do Brasil: descrição de casos. Semina Ciênc. Agrar. 24(1):171-176.

ESBÉRARD, C.E.L. 2003. Diversidade de morcegos em área de Mata Atlântica regenerada no Sudeste do Brasil. Rev. Bras. Zoo. 5(2):189-204.

FARIA, D. \& BAUMGARTEN, J. 2007. Shade cacao plantations (Theobroma (acao) and bat conservation in southern Bahia, Brazil. Biodivers. Conserv. 16:291-312. 
FENTON, M.B., ACHARYA, L., AUDET, D., HICKEY, M.B.C., MERRIMAN, C., OBRIST, M.K. \& SYME, D.M. 1992. Phyllostomid bats (Chiroptera: Phyllostomidae) as indicators of habitat disruption in the Neotropics. Biotropica 24(3):440-446.

FISCHER, E. 1992. Foraging of Nectarivorous Bats on Bauhinia ungulata. Biotropica 24:579-582.

FISCHER, W.A. \& FISCHER, E.A. 1995. Comportamento social e reprodutivo do morcego-cara-branca, Artibeus lituratus. In Ecologia e preservação de uma floresta tropical urbana (H.F.L. Filho \& L.P.C. Morellato, Org.). Editora da UNICAMP, Campinas, SP.

FISCHER, E., MUNIN, R.L., LONGO, J.M., FISCHER, W.A. \& SOUZA, P.R. 2010. Predation on bats by Great Kiskadees. J. Field Ornithol. 81:17-20.

FLEMING, T.H. 1992. How do fruit- and nectar-feeding birds and Mammals track their food resources? In Effects of resource distribution on animalplant interactions (M.D. Hunter, T. Ohgushi \& P.W. Price, Ed.). Academic Press, p.355-391.

FLEMING, T., HOOPER, E. \& WILSON, D. 1972. Three Central American bat communities: structure reproductive cycles, and movement patterns. Ecology 53:555-569.

GREGORIN, R. \& TADDEI, V.A. 2002. Chave artificial para identificação de molossideos brasileiros (Mammalia Chiroptera). J. Neotrop. Mammal. 9(1):13-32.

GRUENER, C.G. 2006. Efeito da fragmentação florestal sobre as comunidades de morcegos do município de Blumenau, SC. Dissertação, Universidade Regional de Blumenau, Blumenau.

HAMMER, Ø., HARPER, D.A.T. \& RYAN, P.D. 2001. PAST: Paleontological Statistics Software Package for Education and Data Analysis. Palaeontologia Eletronica, 4. http://palaeo-electronica.org/2001_1/past/ issue1_01.htm (último acesso em 07/02/2010).

INSTITUTO MUNICIPAL DE PLANEJAMENTO URBANO - PLANURB. 2007. Perfil Sócio-Econômico de Campo Grande Estado do Mato Grosso do Sul.

JEPSON, W.A. 2005. A disappearing biome? reconsidering land-cover change in the Brazilian savanna. Geogr. J. 171:99-111.

KLINK, C.A. \& MACHADO, R.B. 2005. A conservação do Cerrado brasileiro. Megadiversidade 1:147-155.

KUNZ, T.H., AUGUST, P.V. \& BURNETT, C.D. 1983. Harem social organization in cave roosting Artibeus jamaicensis (Chiroptera: Phyllostomidae). Biotropica 15(2):133-138.

KWIECINSKI, G.G. 2006. Phyllostomus discolor. Mammalian Species 801:1-11.

LIMA, I.P. 2008. Espécies de morcegos (Mammalia, Chiroptera) registradas em parques nas áreas urbanas do Brasil e suas implicações no uso deste ambiente. In Ecologia de morcegos (N.R. Reis, A.L. Peracchi \& G.A.S.D. Santos, Org.). Technnical books, Londrina, p.71-85.

MAGURRAN, A.E. 2004. Measuring biological diversity. Blackwell Publishing, Oxford, p.1-71.

MELLO, M.A.R. \& FERNANDEZ, F.A.S. 2000. Reproductive ecology of bat Carollia perspicillata (Chiroptera: Phyllostomidae) in a fragment of the Brazilian Atlantic coastal forest. Z. Saeugetierkd 65:340-349.

MELLO, M.A.R., KALKO, E.K.V. \& SILVA, W.R. 2009. Ambient temperature is more important than food availability in explaining reproductive timing of the bat Sturnira lilium (Mammalia: Chiroptera) in a montane Atlantic Forest. Can. J. Zool. 87:239-245.

MORAIS, A.I.F. 2002. Inventário da quiropterofauna da área urbana de Cuiabá - MT: subsídios para conservação e manejo. Dissertação, Universidade Federal de Mato Grosso, Cuiabá.

MYERS, N., MITTERMEIER, R.A., MITTERMEIER, C.G., FONSECA, G.A.B. \& KENT, J. 2000. Biodiversity hotspots for conservation priorities. Nature 403:853-858.

NOGUEIRA, M.R. \& PERACCHI, A.L. 2003. Fig-seed predation by 2 species of Chiroderma: discovery of a new feeding strategy in bats. J. Mammal. 84(1):225-233.
OPREA, M., MENDES, P., VIEIRA, T.B. \& DITCHFIELD, A.D. 2009. Do wooded streets provide connectivity for bats in an urban landscape? Biodivers. Conserv. 18:2361-2371.

ORTÊNCIO-FILHO, H., REIS, N.R., PINTO, D., ANDERSON, R., APARECIDA, D. \& MARQUES, M.A. 2005. Levantamento dos morcegos (Chiroptera, Mammalia) do Parque Municipal do Cinturão Verde de Cianorte, Paraná, Brasil. Chiropt. Neotrop. 11:1-2.

PEDRO, W.A. \& TADDEI, V.A. 1997. Taxonomic assemblage of bats from Panga Reserve, Southeastern Brazil: abundance patterns and trophic relations in the Phyllostomidae (Chiroptera). Bol. Mus. Biol. Mello Leitão 6:3-21.

PERINI, F.A., TAVARES, V.C. \& NASCIMENTO, C.M.D. 2003. Bats from the city of Belo Horizonte, Minas Gerais, Southeastern Brazil. Chiropt. Neotrop. 9:1-2.

PULCHÉRIO-LEITE, A. 2008. Uso do espaço por Artibeus lituratus e Sturnira lilium (Chiroptera: Phyllostomidae) em fragmentos florestais urbanos de Curitiba, Paraná. Tese, Universidade Federal do Paraná, Paraná.

PULCHÉRIO-LEITE, A., MENEGHELLI, M. \& TADDEI, A.V. 1999. Morcegos da região de Campo Grande, Estado do Mato Grosso do Sul, com ênfase para as espécies urbanas. Ensaios e Ci. 3:113-129.

PURVIS, A., GITTLEMAN, J.L., COWLISHAW, G. \& MACE, G.M. 2000. Predicting extinction risk in declining species. Proc. R. Soc. Lond. B267:1947-1952.

RACEY, P.A. 1988. Reproductive assessment in bats. In Ecological and behavioral methods for the study of bats (T.H. Kunz, ed). Smithsonian Institution, Washington, p.31-46.

REIS, N.R., LIMA, I.P. \& PERACCHI, A.L. 2002. Morcegos (Chiroptera) da área urbana de Londrina Paraná - Brasil. Rev. Bras. Zool. 19(3):739-746.

REIS, N.R., PERACCHI, A.L., PEDRO, W.A. \& LIMA, I.P. 2007. Morcegos do Brasil. Londrina, 253p.

SANO, E.E., ROSA, R., BRITO, J.L.S. \& FERREIRA, L.G. 2007. Mapeamento de cobertura vegetal do Bioma Cerrado: estratégias e resultados. Embrapa Cerrados, Planaltina, p.33.

SANTOS, M., AGUIRRE, L.F., VÁQUEZ, L.B. \& ORTEGA, J. 2003. Phyllostomus hastatus. Mamm. Species 722:1-6.

SAZIMA, I., FISCHER, W.A., SAZIMA, M. \& FISCHER, E. 1994. The fruit bat Artibeus lituratus as a forest and city dweller. Ciênc. Cult. 46(3):164-168.

SILVA, M.M.S., HARMANI, N.M.S., GONÇALVES, E.F.B. \& UIEDA, W. 1996. Bats from the metropolitan region of São Paulo, Southeastern Brazil. Chiropt. Neotrop. 2:39-41.

STEVENS, R.D. \& WILLIG, M.R. 2000. Community, abundance and morphology. Oikos 88:48-56.

STRAUBE, F.C. \& BIANCONI, G.V. 2002. Sobre a grandeza e a unidade utilizada para estimar esforço de captura com utilização de redes-deneblina. Chiropt. Neotrop. 8:1-2.

STUTZ, W.H., ALBUQUERQUE, M.C., UIEDA, W., MACEDO, E.M. \& FRANÇA, C.B. 2004. Updated list of Uberlândia bats (Minas Gerais State, Southeastern Brazil). Chiropt. Neotrop. 10: 1-2.

TEIXEIRA, R.C., CORRÊA, C.E. \& FISCHER, E. 2009. Frugivory by Artibeus jamaicensis (Phyllostomidae) bats in the Pantanal, Brazil. Stud. Neotrop. Fauna E. 44:7-15.

VIZOTTO, L.D. \& TADDEI, V.A. 1973. Chave para a identificação de quirópteros brasileiros. Bol. Ciências 1:1-72.

ZORTÉA, M. 2003. Reproductive patterns and feeding habits of three nectarivorous bats (Phyllostomidae: Glossophaginae) from de Brazilian Cerrado. Braz. J. Biol. 63(1):159-168.

ZORTÉA, M. \& CHIARELLO, A.G. 1994. Observations on the big fruiteating bat, Artibeus lituratus, in an urban reserve of south-east Brazil. Mammalia 58:665-670. 